\title{
Investigation of manganese homeostasis in dogs with anaemia and chronic enteropathy
}

\author{
Marisa da Fonseca Ferreira*, Arielle Elizabeth Ann Aylor, Richard John Mellanby, Susan Mary Campbell and \\ Adam George Gow \\ Hospital for Small Animals, The Royal (Dick) School of Veterinary Studies, The University of Edinburgh, UK
}

\begin{abstract}
Lethargy is a frequent and important clinical feature of anaemia; however, it does not absolutely correlate with the severity of anaemia. Manganese is efficiently absorbed through the gastrointestinal tract via divalent metal transporter 1 (DMT1), which is also responsible for iron transport. DMT1 is upregulated in iron deficiency (ID). Increased manganese concentrations are reported in ID anaemia (IDA) in various species. Manganese is neurotoxic and therefore may contribute to lethargy observed in some anaemic patients. In addition, anaemia and ID are common in human inflammatory bowel disease. Little is known about how anaemia influences manganese metabolism in veterinary patients and how common is anaemia in dogs with chronic enteropathy (CE). If elevated manganese concentrations are found, then potentially neurotoxicity may be contributing to morbidity in these cases. The objectives of this study were to investigate the hypothesis that whole blood manganese concentrations would be increased in dogs with anaemia, particularly in dogs with confirmed IDA, and that anaemia would be common in canine CE. Medical records from 2012-2016 were reviewed for dogs with CE that were anaemic, as well as dogs with confirmed IDA, where a sample suitable for manganese analysis was held in an archive. Manganese concentration was measured in whole blood from: 11 anaemic dogs with CE, 6 dogs with IDA, 9 non-anaemic ill controls, and 12 healthy controls. MannWhitney U and Kruskal-Wallis tests with post-test Dunn's multiple comparisons tests were performed, with $P<0.05$ considered significant. The prevalence of anaemia in canine $\mathrm{CE}$ was $20.6 \%$ (33/160). Manganese concentrations were significantly different between all groups $(P=0.0001)$ and higher in non-anaemic than anaemic $\operatorname{dogs}(P=0.0078)$. Manganese concentrations were also higher in healthy compared to ill controls $(P<0.0001)$, anaemic dogs with CE $(P=0.0056)$ and to dogs with IDA $(P=0.0001)$. No differences were observed between anaemic dogs with CE, IDA and ill controls. Although anaemia was frequently observed in canine CE, the hypothesis that dogs with anaemia would have increased manganese concentrations, possibly contributing to a lethargic state was not supported. Further research is warranted to understand the influence of anaemia on whole blood manganese.
\end{abstract}

Keywords: Inflammatory bowel disease, Iron, Trace element.

\section{Introduction}

Lethargy is a common clinical sign in anaemia. Although the reduction in haemoglobin concentration results in decreased delivery of oxygen to tissues, lethargy does not always correlate with anaemia severity (Chervier et al., 2012; Bager, 2014). Therefore, it is possible that other factors might be contributing to lethargy. In humans with iron deficiency anaemia (IDA), increased intestinal iron absorption also results in increased absorption of other trace elements including manganese (Mn) (Meltzer et $a l ., 2010)$. Manganese is an essential trace element and a necessary cofactor for a few enzymes and metabolic pathways. Manganese is efficiently absorbed from the gastrointestinal tract via divalent metal transporter 1 (DMT1), which is concurrently responsible for iron transport (Au et al., 2008).

Iron deficiency (ID) causes upregulation of DMT1 and has been associated with increased Mn concentrations in many species (Kim and Lee, 2011; Smith et al., 2013).

Manganese is neurotoxic when present in excessive concentrations (Racette et al., 2017). The mechanism of neurotoxicity appears multifactorial. Astrocytic dysfunction occurs due to their high affinity transport system accumulating high intracellular concentrations of Mn (Yin et al., 2008). Manganese also causes microglial activation by induction of cytokines and reactive oxygen species (Dodd and Filipov, 2011), as well as causing disruption of several neurotransmitters and mitochondrial dysfunction, culminating in neuronal apoptosis (Smith et al., 2017).

Hyperintensity of the basal ganglia on magnetic resonance imaging (MRI) is pathognomonic for $\mathrm{Mn}$ deposition in humans, correlating with neurological signs and fatigue in human cirrhosis as well as blood Mn concentrations (Burkhard et al., 2003; Forton et al., 2004). Hyperintensity in focal areas of the basal ganglia

*Corresponding Author: Marisa da Fonseca Ferreira. Easter Bush Campus, Roslin, EH25 9RG, UK. Tel.: +441316507650; 
has also been shown in canine and feline congenital portosystemic shunts, which contained increased concentrations of $\mathrm{Mn}$ on post-mortem examination (Torisu et al., 2008).

Iron deficiency has been linked with Mn accumulation within the brain, and increased uptake by astrocytes, mediated by DMT1 upregulation, in the absence of Mn overexposure (Erikson and Aschner, 2006). The presence of clinical Mn neurotoxicity with concurrent ID, and without Mn exposure, has been suggested in developing mice and confirmed in one child (KwikUribe et al., 1999; Brna et al., 2011).

Anaemia is one of the most common extra-intestinal clinicopathological abnormalities in human inflammatory bowel disease (IBD), associated with a multifactorial aetiology, however over half of the cases demonstrated ID (Filmann et al., 2014). Notably, ID is suggested to affect quality of life in human IBD, even in the absence of anaemia (Herrera-deGuise et al., 2016).

There are only a few studies examining Mn homeostasis in human IBD. One report demonstrated higher whole blood Mn levels in 5/55 patients with ulcerative colitis, not associated with clinical or MRI evidence of toxicity, with $2 / 5$ cases confirming low ferritin levels (El Muhtaseb et al., 2007). The remaining studies have shown no differences, though all reports were based in serum or plasma Mn (Whineray et al., 2000; Ma et al., 2013). More importantly, none have concentrated specifically on anaemic, or iron deficient subjects.

Chronic enteropathies (CE) are a common cause of morbidity and mortality in dogs (Allenspach et al., 2016). Metabolic complications are well described in dogs with CE, notably hypoalbuminaemia, hypocobalaminaemia and altered vitamin D status (Allenspach et al., 2007; Gow et al., 2011). Canine CE appears to share similarities to human IBD (Cerquetella et al., 2010). Surprisingly, studies characterising anaemia in canine $\mathrm{CE}$ are scant, although a prevalence is reported of between $12-18 \%$ in studies with relatively small numbers (Craven et al., 2004; Marchetti et al., 2010). Essential trace elements in canine CE are even less well studied, with only one abstract reporting plasma Mn concentrations, showing no difference compared to laboratory beagle controls (Yokoyama et al., 2016).

Given the high prevalence of anaemia in human IBD, the aim of this study was to investigate the prevalence of anaemia in a larger number of cases of canine CE, and to then establish whether anaemia is associated with increased Mn concentrations. An additional aim was to assess if confirmed absolute IDA, regardless of underlying aetiology, is associated with higher Mn concentrations. Non-anaemic controls, both ill and healthy, served as cohorts for comparison.

\section{Materials and Methods}

\section{Samples}

The small animal teaching hospital archive, at the authors' institution, was used to retrieve the samples for the study. The archive database of $-80^{\circ} \mathrm{C}$ stored residual samples previously taken for clinical diagnostic purposes, with informed consent from the owners, was searched for ethylenediaminetetraacetic acid (EDTA) samples from January 2012 to January 2016.

\section{Inclusion criteria}

Using the corresponding patients' case numbers, a retrospective search using the hospital's electronic record system was undertaken to retrieve clinical information that would match inclusion criteria for each group: anaemic dogs with a clinical diagnosis of CE, either food responsive, antibiotic responsive or idiopathic, confirmed histologically from endoscopic or full thickness surgical gastric and/or intestinal biopsies; dogs with absolute IDA; ill dogs with normal haematology; and healthy dogs based on history and physical examination, with a normal packed cell volume (PCV). Mn analysis was performed on small cohorts of the first and last two groups, and on all patients with confirmed IDA.

\section{Analyses}

Gastrointestinal histology and haematology analyses were performed by the institution's pathology laboratory, with results confirmed by board-certified veterinary pathologists and clinical pathologists, respectively. Histological results confirming a diagnosis of CE would need to include mild, moderate or marked mucosal infiltration by inflammatory cells (lymphocytes, plasma cells, eosinophils). Anaemia was defined by a PCV below the lower limit of the laboratory reference range $(0.39 \mathrm{~L} / \mathrm{L})$. Iron status analyses were performed by an external laboratory and diagnosis of absolute ID was defined by serum iron below the reference interval, combined with transferrin saturation below the reference interval and a normal total iron binding capacity (TIBC).

Manganese concentrations were prospectively determined at an external laboratory, by graphite furnace atomic absorption spectrometry (1100 Spectrometer, PerkinElmer Life and Analytical Sciences, Milan, Italy), in whole blood anticoagulated with EDTA, after dilution with Triton X-100 solution (Sigma-Aldrich, St. Louis, MO). All samples had been frozen within 4 hours of collection, archived as abovementioned, and subsequently shipped in dry ice to the reference laboratory. The assay had a limit of quantitation of $16 \mathrm{nmol} / \mathrm{L}$, an inter-assay coefficient of variation $(\mathrm{CV})$ of $3.1 \%$ and an intra-assay $\mathrm{CV}$ of $5.4 \%$.

\section{Statistical analysis}

Assessment for normality was performed with the Kolmogorov-Smirnov test for each group and the overall population regarding $\mathrm{Mn}$ concentrations, PCV 
and age. As not all groups were normally distributed in each of the three categories, nonparametric tests were therefore used for statistical analysis throughout, with data expressed as median (minimum/maximum ranges). Mann-Whitney $U$ and Kruskal-Wallis tests with post-test Dunn's multiple comparisons tests were performed for assessment of differences in between groups. The correlation between Mn concentration and PCV was assessed by the Spearman's rank correlation coefficient $\left(\mathrm{r}_{\mathrm{s}}\right)$. Statistical significance level was set at $P<0.05$. Statistical analysis was performed with two commercial software packages (GraphPad InStat, version 3.10, GraphPad Software Inc.; and Minitab®, version 17.1.0, Minitab Inc.).

\section{Results}

A total of 1847 whole blood EDTA samples were available, from which $160(8.7 \%)$ corresponded to dogs with histologically confirmed CE. Of these, anaemia was present in 33 (20.6\%), with one patient being microcytic and hypochromic, and four normocytic and hypochromic. A cohort of the most anaemic dogs with $\mathrm{CE}(\mathrm{n}=11)$ was selected for Mn analysis, of which four had iron status results available, confirming absolute IDA in two, and anaemia of chronic disease (ACD) with relative IDA (low serum iron with normal transferrin saturation and low TIBC) in another two.

Within the archived available whole blood EDTA samples, six corresponded to cases from which an iron status confirmed absolute ID. Of these, two dogs were in the previously selected CE group, and the remaining had an underlying diagnosis each of: intestinal lymphoma, immune-mediated haemolytic anaemia, urinary bladder mass, or suspected disseminated histiocytic neoplasia.

Including the non-anaemic ill $(\mathrm{n}=9$, Table 1$)$ and healthy $(n=12)$ cohort controls, a total of 36 samples were then prospectively analysed for Mn concentration. No differences were seen between groups with regard to age $(P=0.2025)$ or gender $(P=0.1025)$. Eight individuals were crossbreeds and the most commonly represented breeds included: Labrador Retriever $(\mathrm{n}=$ $6)$, Cocker Spaniel $(n=4)$, followed by Boxer $(n=2)$, German Shepherd $(n=2)$, Hungarian Vizla $(n=2)$ and Springer Spaniel $(n=2)$. Mn concentrations and PCVs in each group are summarised in Figure 1 and Table 2. Manganese concentrations were significantly different between the four groups $(P=0.0001)$, being higher in healthy dogs compared to: anaemic dogs with CE $(P=$ $0.0056)$, dogs with IDA $(P=0.0001)$, and non-anaemic ill controls $(P<0.0001)$. No differences were observed between Mn concentrations in anaemic dogs with CE, dogs with IDA and non-anaemic ill controls. Mn concentrations were also higher in overall non-anaemic compared to anaemic dogs $(P=0.0078)$, with an overall moderate positive correlation between PCV and $\mathrm{Mn}$ concentration $\left(\mathrm{r}_{\mathrm{s}}=0.6252, P<0.0001\right)$.
Table 1. Problem list/diagnoses of a cohort of non-anaemic ill dogs $(n=9)$.

\begin{tabular}{lc}
\hline Diagnosis & Patients (n) \\
\hline Chronic cystitis & 2 \\
\hline Chronic intermittent diarrhoea & 2 \\
\hline Acute gastroenteritis & 1 \\
\hline $\begin{array}{l}\text { Cervical carcinoma, prostatic mass } \\
\text { Hepatosplenic nodules, gastric mass, } \\
\text { chronic kidney disease }\end{array}$ & 1 \\
Septic peritonitis of unknown origin & 1 \\
\hline Sinonasal aspergillosis & 1 \\
\hline
\end{tabular}

Table 2. Descriptive statistics of manganese concentrations and packed cell values distribution within four cohorts.

\begin{tabular}{lcccc}
\hline & \multicolumn{2}{c}{ Mn $(\mathrm{nmol} / \mathrm{L})$} & \multicolumn{2}{c}{ PCV $(\mathrm{l} / \mathrm{l})$} \\
\cline { 2 - 5 } Cohort & Median & Range & Median & Range \\
\hline Anaemic CE & 366 & $219-1817$ & 0.27 & $0.13-0.32$ \\
IDA & 292 & $170-710$ & 0.20 & $0.13-0.30$ \\
$\begin{array}{l}\text { Non- } \\
\text { anaemic ill }\end{array}$ & 608 & $303-839$ & 0.46 & $0.42-0.51$ \\
$\begin{array}{l}\text { Healthy } \\
\text { Henc }\end{array}$ & 1006 & $893-1240$ & 0.53 & $0.45-0.64$ \\
\hline
\end{tabular}

Footnote: CE - chronic enteropathy; IDA - iron-deficiency anaemia; $\mathrm{Mn}$ - manganese; $\mathrm{PCV}$ - packed cell value.

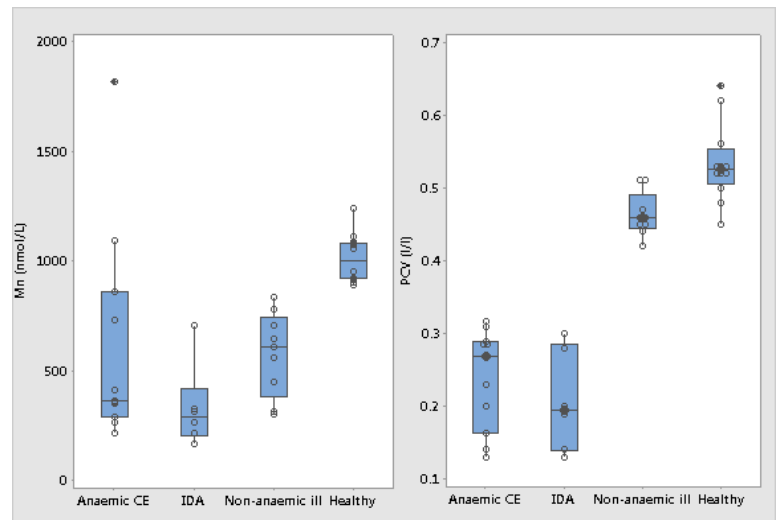

Fig. 1. Box and whisker plots and individual values depicting manganese (Mn) concentrations (left) and packed cell values (PCV, right) distribution within four cohorts. (CE): Chronic enteropathy; (IDA): Iron-deficiency anaemia.

However, when analysing healthy dogs alone, there was no correlation between PCV and Mn $\left(\mathrm{r}_{\mathrm{s}}=-11.35\right.$, $P=0.7329)$. Moreover, when comparing PCV overall amongst groups, the difference obtained $(P<0.0001)$ was attributed only to the comparison between each of the anaemic groups (dogs with CE or ID), with each of the non-anaemic ill or healthy control groups, and no difference was seen in between the latter two cohorts on post-test Dunn's multiple comparisons test. 


\section{Discussion}

Anaemia in canine CE was frequent within the population studied. The obtained prevalence of $20.6 \%$ was higher than previously reported, as was the overall number of patients assessed, totalling 160, compared with 22 and 77 in other studies (Craven et al., 2004; Marchetti et al., 2010). This prevalence is similar to that of $24 \%$ obtained in a meta-analysis in human IBD (Filmann et al., 2014). This confirms that anaemia is a common clinicopathological abnormality in canine CE and may be contributing towards overall morbidity.

The results obtained in this study showed no evidence that ID is associated with increased whole blood Mn concentrations. Moreover, anaemic dogs with $\mathrm{CE}$ or IDA, as well as non-anaemic ill dogs, had lower Mn concentrations when compared to healthy controls. This does not support the hypothesis that $\mathrm{Mn}$ neurotoxicity contributes to the clinical sign of lethargy in these cases.

There are many potential factors which may explain this result. As the majority of circulating $\mathrm{Mn}$ is within erythrocytes, it is possible that anaemia may be a confounding factor, although no correlation between $\mathrm{Mn}$ and PCV was found in the healthy controls (Pleban and Pearson, 1979).

Measurement of whole blood $\mathrm{Mn}$ is recommended to reflect whole body manganese (Clegg et al., 1986). Whole blood $\mathrm{Mn}$ is less variable within an individual, when compared to plasma, better reflecting $\mathrm{Mn}$ in a single sample (Baker et al., 2015). A higher risk of inaccuracy is seen with serum measurements, combined with wider variation of reference ranges (Baruthio et al., 1988). Even if no macroscopic haemolysis is present, there can be a considerable amount of Mn that is released from the red blood cells into the serum or plasma if not separated, leading to potential erroneous interpretations. In addition, to be reliable, serum or plasma analysis requires very sensitive assays, since $\mathrm{Mn}$ concentration is approximately 10-30 times lower than within erythrocytes (Versieck et al., 1974).

Moreover, other studies have used whole blood samples to assess Mn status in ID and were able to confirm increased $\mathrm{Mn}$ concentrations in anaemic samples (Meltzer et al., 2010; Kim and Lee, 2011; Smith et al., 2013).

Whole blood manganese is obtained from dietary intake. These clinical cases were on a range of diets, which would likely have widely different Mn content, as previously documented on a comparison of several commercial diets (Gagne et al., 2013). Hyporexia is a common finding in any illness, and one of the main clinical signs of canine CE (Gianella et al., 2017), therefore reduced dietary intake is a potential confounding factor for both the non-anaemic ill and CE groups. The healthy control group dogs were all fed a standard complete diet with a Mn content of $25.1 \mathrm{mg} / \mathrm{kg}$ on a dry matter basis, which is within the minimum and maximum amounts $(5.8 \mathrm{mg} / \mathrm{kg}$ and $170 \mathrm{mg} / \mathrm{kg}$, respectively) recommended for adult dogs, by the European Pet Food Industry Federation (Zentek, 2016). It is possible that this standardised diet may explain the higher concentrations seen in the healthy group in this study.

Inflammation seen in canine $\mathrm{CE}$ might stimulate hepcidin, a peptide produced in the liver, controlling iron homeostasis by inhibiting iron transport (BrasseLagnel et al., 2011). This hormone downregulates DMT1 and also binds to the iron and Mn exporter ferroportin 1, leading to its internalisation and degradation, resulting in iron (and likely $\mathrm{Mn}$ ) entrapment and decreased transport across the basolateral membrane (Seo and Wessling-Resnick, 2015). Hepcidin has been shown to be upregulated in human IBD, resulting in ACD due to relative ID (Bergamaschi et al., 2013). As a further variable, Vitamin D is an important suppressor of hepcidin, and dogs with severe CE have been shown to have reduced vitamin D concentrations (Gow et al., 2011; Bacchetta et al., 2014). Moreover, in inflammatory conditions, the action of metal transporters can be disrupted, independently of hepcidin (Guida et al., 2015). In human IBD, active disease was seen to correlate with decreased mucosal DMT1 expression (Wu et al., 2015). Conversely, in a study of human IBD, where 14/19 patients were anaemic while in histological remission, upregulation of DMT1 was confirmed, and negatively correlated with haemoglobin, possibly explained by absolute ID overriding the effects of ACD (Sukumaran et al., 2014). Investigation of DMT1 expression in enterocytes in canine $\mathrm{CE}$ would help define this effect. In addition, is unknown how the presence of other iron and Mn transporters, namely ZIP8 and ZIP14, might influence overall canine Mn homeostasis (Shawki et al., 2015). Given that dogs with gastrointestinal disease (CE, lymphoma, and non-specified) were present within the absolute IDA and non-anaemic ill groups (n $=3$ in each group), is possible that impaired $\mathrm{Mn}$ absorption in these patients has accounted for the lower concentrations observed, alongside the anaemic CE group.

Finally, the liver is responsible for removing most of the Mn absorbed through the gastrointestinal tract from the portal circulation, excreting it via the biliary system and allowing only around $2 \%$ of the absorbed Mn to reach the systemic circulation (Papavasiliou et al., 1966; Klaassen, 1974). This system is very efficient and dogs fed large quantities of Mn over many weeks demonstrated no significant increase in whole blood manganese concentrations, yet had increased hepatic concentrations, thought to be due to trafficking through the liver for excretion in bile (Reiman and Minot, 
1920). Therefore, it is possible that despite increased Mn delivery in the portal vasculature, the canine liver is more efficient at Mn extraction, leading to no systemic increase. Dogs with hepatic dysfunction and portosystemic shunting have altered Mn homeostasis, leading to Mn accumulation (Kilpatrick et al., 2014). Consequently, further research would be needed to assess if ID could play a role in exacerbating increased Mn concentrations in dogs with established liver pathology, as reported in human chronic liver disease (Malecki et al., 1999).

This study carries several limitations. Although Mn levels were prospectively measured, the population was retrospectively assessed, thereby leading to lack of standardisation within and between groups. The number of dogs in each group was also small, which could have led to subsequent type II error regarding the absence of difference between anaemic and nonanaemic ill dogs. Iron status analysis was only available for eight subjects, precluding further conclusions. In addition, diet was not standardised, which could have affected results (Lopez-Alonso et al., 2007).

This study demonstrates that anaemia is common in dogs with chronic enteropathy. However, anaemic dogs with chronic enteropathy and/or iron-deficiency anaemia do not have increased whole blood manganese concentrations, compared to non-anaemic ill or healthy cohorts. More investigation is warranted to understand the complex interplay of canine anaemia, iron deficiency and inflammatory disease, namely chronic enteropathy, in manganese concentrations.

\section{Acknowledgments}

Manganese analyses were reported by Antony Catchpole, Scottish Trace Element \& Micronutrient Diagnostic \& Research Laboratory, North Glasgow Biochemistry Department, NHS Great Glasgow and Clyde, United Kingdom.

No external funds were used for this study.

\section{Conflict of interest}

The authors declare that there is no conflict of interest.

\section{References}

Allenspach, K., Culverwell, C. and Chan, D. 2016. Long-term outcome in dogs with chronic enteropathies: 203 cases. Vet. Rec. 178(15), 368.

Allenspach, K., Wieland, B., Grone, A. and Gaschen, F. 2007. Chronic enteropathies in dogs: evaluation of risk factors for negative outcome. J. Vet. Intern. Med. 21(4), 700-708.

$\mathrm{Au}$, C., Benedetto, A. and Aschner, M. 2008. Manganese transport in eukaryotes: the role of DMT1. Neurotoxicology 29(4), 569-576.

Bacchetta, J., Zaritsky, J.J., Sea, J.L., Chun, R.F., Lisse, T.S., Zavala, K., Nayak, A., Wesseling-Perry, K., Westerman, M., Hollis, B.W., Salusky, I.B. and Hewison, M. 2014. Suppression of iron-regulatory hepcidin by vitamin D. J. Am. Soc. Nephrol. 25(3), 564-572.

Bager, P. 2014. Fatigue and acute/chronic anaemia. Dan. Med. J. 61(4), B4824.

Baker, M.G., Simpson, C.D., Sheppard, L., Stover, B., Morton, J., Cocker, J. and Seixas, N. 2015. Variance components of short-term biomarkers of manganese exposure in an inception cohort of welding trainees. J. Trace Elem. Med. Biol. 29, 123-129.

Baruthio, F., Guillard, O., Arnaud, J., Pierre, F. and Zawislak, R. 1988. Determination of manganese in biological materials by electrothermal atomic absorption spectrometry: a review. Clin. Chem. 34(2), 227-234.

Bergamaschi, G., Di Sabatino, A., Albertini, R., Costanzo, F., Guerci, M., Masotti, M., Pasini, A., Massari, A., Campostrini, N., Corbella, M., Girelli, D. and Corazza, G.R. 2013. Serum hepcidin in inflammatory bowel diseases: biological and clinical significance. Inflamm. Bowel Dis. 19(10), 2166-2172.

Brasse-Lagnel, C., Karim, Z., Letteron, P., Bekri, S., Bado, A. and Beaumont, C. 2011. Intestinal DMT1 cotransporter is down-regulated by hepcidin via proteasome internalization and degradation. Gastroenterology 140(4), 1261-1271 e1261.

Brna, P., Gordon, K., Dooley, J.M. and Price, V. 2011. Manganese toxicity in a child with iron deficiency and polycythemia. J. Child Neurol. 26(7), 891-894.

Burkhard, P.R., Delavelle, J., Du Pasquier, R. and Spahr, L. 2003. Chronic parkinsonism associated with cirrhosis: a distinct subset of acquired hepatocerebral degeneration. Arch. Neurol. 60(4), 521-528.

Cerquetella, M., Spaterna, A., Laus, F., Tesei, B., Rossi, G., Antonelli, E., Villanacci, V. and Bassotti, G. 2010. Inflammatory bowel disease in the dog: differences and similarities with humans. World J. Gastroenterol. 16(9), 1050-1056.

Chervier, C., Cadore, J.L., Rodriguez-Pineiro, M.I., Deputte, B.L. and Chabanne, L. 2012. Causes of anaemia other than acute blood loss and their clinical significance in dogs. J. Small Anim. Pract. 53(4), 223-227.

Clegg, M.S., Lonnerdal, B., Hurley, L.S. and Keen, C.L. 1986. Analysis of whole blood manganese by flameless atomic absorption spectrophotometry and its use as an indicator of manganese status in animals. Anal. Biochem. 157(1), 12-18.

Craven, M., Simpson, J.W., Ridyard, A.E. and Chandler, M.L. 2004. Canine inflammatory bowel disease: retrospective analysis of diagnosis and outcome in 80 cases (1995-2002). J. Small Anim. Pract. 45(7), 336-342.

Dodd, C.A. and Filipov, N.M. 2011. Manganese potentiates LPS-induced heme-oxygenase 1 in 
microglia but not dopaminergic cells: role in controlling microglial hydrogen peroxide and inflammatory cytokine output. Neurotoxicology 32(6), 683-692.

El Muhtaseb, M.S., Duncan, A., Talwar, D.K., O'Reilly, D.S., McKee, R.F., Anderson, J.H. and Finlay, I.G. 2007. Assessment of dietary intake and trace element status in patients with ileal pouch-anal anastomosis. Dis. Colon. Rectum. 50(10), 15531557.

Erikson, K.M. and Aschner, M. 2006. Increased manganese uptake by primary astrocyte cultures with altered iron status is mediated primarily by divalent metal transporter. Neurotoxicology 27(1), 125-130.

Filmann, N., Rey, J., Schneeweiss, S., Ardizzone, S., Bager, P., Bergamaschi, G., Koutroubakis, I., Lindgren, S., Morena Fde, L., Moum, B., Vavricka, S.R., Schroder, O., Herrmann, E. and Blumenstein, I. 2014. Prevalence of anemia in inflammatory bowel diseases in european countries: a systematic review and individual patient data meta-analysis. Inflamm. Bowel Dis. 20(5), 936-945.

Forton, D.M., Patel, N., Prince, M., Oatridge, A., Hamilton, G., Goldblatt, J., Allsop, J.M., Hajnal, J.V., Thomas, H.C., Bassendine, M., Jones, D.E. and Taylor-Robinson, S.D. 2004. Fatigue and primary biliary cirrhosis: association of globus pallidus magnetisation transfer ratio measurements with fatigue severity and blood manganese levels. Gut 53(4), 587-592.

Gagne, J.W., Wakshlag, J.J., Center, S.A., Rutzke, M.A. and Glahn, R.P. 2013. Evaluation of calcium, phosphorus, and selected trace mineral status in commercially available dry foods formulated for dogs. J. Am. Vet. Med. Assoc. 243(5), 658-666.

Gianella, P., Lotti, U., Bellino, C., Bresciani, F., Cagnasso, A., Fracassi, F., D'Angelo, A. and Pietra, M. 2017. Clinicopathologic and prognostic factors in short- and long-term surviving dogs with proteinlosing enteropathy. Schweiz. Arch. Tierheilkd. 159(3), 163-169.

Gow, A.G., Else, R., Evans, H., Berry, J.L., Herrtage, M.E. and Mellanby, R.J. 2011. Hypovitaminosis D in dogs with inflammatory bowel disease and hypoalbuminaemia. J. Small Anim. Pract. 52(8), 411-418.

Guida, C., Altamura, S., Klein, F.A., Galy, B., Boutros, M., Ulmer, A.J., Hentze, M.W. and Muckenthaler, M.U. 2015. A novel inflammatory pathway mediating rapid hepcidin-independent hypoferremia. Blood 125(14), 2265-2275.

Herrera-deGuise, C., Casellas, F., Robles, V., Navarro, E. and Borruel, N. 2016. Iron Deficiency in the Absence of Anemia Impairs the Perception of Health-Related Quality of Life of Patients with
Inflammatory Bowel Disease. Inflamm. Bowel Dis. 22(6), 1450-1455.

Kilpatrick, S., Jacinto, A., Foale, R.D., Tappin, S.W., Burton, C., Frowde, P.E., Elwood, C.M., Powell, R., Duncan, A., Mellanby, R.J. and Gow, A.G. 2014. Whole blood manganese concentrations in dogs with primary hepatitis. J. Small Anim. Pract. 55(5), 241-246.

Kim, Y. and Lee, B.K. 2011. Iron deficiency increases blood manganese level in the Korean general population according to KNHANES 2008. Neurotoxicology 32(2), 247-254.

Klaassen, C.D. 1974. Biliary excretion of manganese in rats, rabbits, and dogs. Toxicol. Appl. Pharmacol. 29(3), 458-468.

Kwik-Uribe, C.L., Golubt, M.S. and Keen, C.L. 1999. Behavioral consequences of marginal iron deficiency during development in a murine model. Neurotoxicol. Teratol. 21(6), 661-672.

Lopez-Alonso, M., Miranda, M., Garcia-Partida, P., Mendez, A., Castillo, C. and Benedito, J.L. 2007. Toxic and trace metal concentrations in liver and kidney of dogs: influence of diet, sex, age, and pathological lesions. Biol. Trace Elem. Res. 116(2), 185-202.

Ma, X., Zhao, K., Wei, L., Song, P., Liu, G., Han, H. and Wang, C. 2013. Altered plasma concentrations of trace elements in ulcerative colitis patients before and after surgery. Biol. Trace Elem. Res. 153(1-3), 100-104.

Malecki, E.A., Devenyi, A.G., Barron, T.F., Mosher, T.J., Eslinger, P., Flaherty-Craig, C.V. and Rossaro, L. 1999. Iron and manganese homeostasis in chronic liver disease: relationship to pallidal T1weighted magnetic resonance signal hyperintensity. Neurotoxicology 20(4), 647-652.

Marchetti, V., Lubas, G., Lombardo, A., Corazza, M., Guidi, G. and Cardini, G. 2010. Evaluation of erythrocytes, platelets, and serum iron profile in dogs with chronic enteropathy. Vet. Med. Int. 2010. Article ID 716040, doi:10.4061/2010/716040.

Meltzer, H.M., Brantsaeter, A.L., Borch-Iohnsen, B., Ellingsen, D.G., Alexander, J., Thomassen, Y., Stigum, H. and Ydersbond, T.A. 2010. Low iron stores are related to higher blood concentrations of manganese, cobalt and cadmium in non-smoking, Norwegian women in the HUNT 2 study. Environ. Res. 110(5), 497-504.

Papavasiliou, P.S., Miller, S.T. and Cotzias, G.C. 1966. Role of liver in regulating distribution and excretion of manganese. Am. J. Physiol. 211(1), 211-216.

Pleban, P.A. and Pearson, K.H. 1979. Determination of manganese in whole blood and serum. Clin. Chem. 25(11), 1915-1918.

Racette, B.A., Searles Nielsen, S., Criswell, S.R., Sheppard, L., Seixas, N., Warden, M.N. and 
Checkoway, H. 2017. Dose-dependent progression of parkinsonism in manganese-exposed welders. Neurology 88(4), 344-351.

Reiman, C.K. and Minot, A.S. 1920. Absorption and elimination of manganese ingested as oxides and silicates. J. Biol. Chem. 45(1), 133-143.

Seo, Y.A. and Wessling-Resnick, M. 2015. Ferroportin deficiency impairs manganese metabolism in flatiron mice. FASEB J. 29(7), 2726-2733.

Shawki, A., Anthony, S.R., Nose, Y., Engevik, M.A., Niespodzany, E.J., Barrientos, T., Ohrvik, H., Worrell, R.T., Thiele, D.J. and Mackenzie, B. 2015. Intestinal DMT1 is critical for iron absorption in the mouse but is not required for the absorption of copper or manganese. Am. J. Physiol. Gastrointest. Liver Physiol. 309(8), G635-647.

Smith, E.A., Newland, P., Bestwick, K.G. and Ahmed, N. 2013. Increased whole blood manganese concentrations observed in children with iron deficiency anaemia. J. Trace Elem. Med. Biol. 27(1), 65-69.

Smith, M.R., Fernandes, J., Go, Y.M. and Jones, D.P. 2017. Redox dynamics of manganese as a mitochondrial life-death switch. Biochem. Biophys. Res. Commun. 482(3), 388-398.

Sukumaran, A., James, J., Janardhan, H.P., Amaladas, A., Suresh, L.M., Danda, D., Jeyeseelan, V., Ramakrishna, B.S. and Jacob, M. 2014. Expression of iron-related proteins in the duodenum is upregulated in patients with chronic inflammatory disorders. Br. J. Nutr. 111(6), 1059-1068.

Torisu, S., Washizu, M., Hasegawa, D. and Orima, H. 2008. Measurement of brain trace elements in a dog with a portosystemic shunt: relation between hyperintensity on T1-weighted magnetic resonance images in lentiform nuclei and brain trace elements. J. Vet. Med. Sci. 70(12), 1391-1393.

Versieck, J., Barbier, F., Speecke, A. and Hoste, J. 1974. Manganese, copper, and zinc concentrations in serum and packed blood cells during acute hepatitis, chronic hepatitis, and posthepatitic cirrhosis. Clin. Chem. 20(9), 1141-1145.

Whineray, E., Inder, W.J., Roche, D., Dobbs, B.R. and Frizelle, F.A. 2000. Comparison of micronutrients in patients having had panproctocolectomy and either ileal pouch anal anastomosis or Brooke ileostomy for chronic ulcerative colitis (UC). Colorectal. Dis. 2(6), 351-354.

Wu, W., Song, Y., He, C., Liu, C., Wu, R., Fang, L., Cong, Y., Miao, Y. and Liu, Z. 2015. Divalent metal-ion transporter 1 is decreased in intestinal epithelial cells and contributes to the anemia in inflammatory bowel disease. Sci. Rep. 5, 16344. doi: 10.1038/srep16344.

Yin, Z., Aschner, J.L., dos Santos, A.P. and Aschner, M. 2008. Mitochondrial-dependent manganese neurotoxicity in rat primary astrocyte cultures. Brain Res. 1203, 1-11.

Yokoyama, N., Ohta, H., Mizukawa, H., Kagawa, Y., Nakayama, S., Sasaki, N., Morishita, K., Nakamura, K., Ikenaka, Y., Ishizuka, M. and Takiguchi, M. 2016. Plasma essential trace element concentrations in dogs with chronic enteropathy. In the Proceedings of the 2016 American College of Veterinary Internal Medicine Forum, pp: 1415.

Zentek, J. 2016. Nutritional guidelines for complete and complementary pet food for cats and dogs. FEDIAF - European Pet Food Industry Federation. 